\title{
An Update on Semisimple Quantum Cohomology and F-Manifolds
}

\author{
Claus Hertling ${ }^{a}$, Yuri I. Manin ${ }^{b, c}$, and Constantin Teleman ${ }^{d, e}$ \\ Received July 2008
}

To the memory of V.A. Iskovskikh

\begin{abstract}
In the first section of this note, we show that Theorem 1.8.1 of Bayer-Manin can be strengthened in the following way: If the even quantum cohomology of a projective algebraic manifold $V$ is generically semisimple, then $V$ has no odd cohomology and is of Hodge-Tate type. In particular, this answers a question discussed by G. Ciolli. In the second section, we prove that an analytic (or formal) supermanifold $M$ with a given supercommutative associative $\mathcal{O}_{M}$-bilinear multiplication on its tangent sheaf $\mathcal{T}_{M}$ is an F-manifold in the sense of HertlingManin if and only if its spectral cover, as an analytic subspace of the cotangent bundle $T_{M}^{*}$, is coisotropic of maximal dimension. This answers a question of V. Ginzburg. Finally, we discuss these results in the context of mirror symmetry and Landau-Ginzburg models for Fano varieties.
\end{abstract}

DOI: $10.1134 /$ S0081543809010088

\section{INTRODUCTION}

Contents of the paper. Semisimple Frobenius manifolds have many nice properties (see, e.g., $[7,14,20,9,10]$ and references therein). It is important to understand as precisely as possible which projective algebraic manifolds $V$ have (generically) semisimple quantum cohomology. In this case the quantum cohomology is determined by initial conditions at one point, a finite amount of numbers, and a mirror (Landau-Ginzburg model) can in many cases be described explicitly.

If $V$ has nontrivial odd cohomology, its full quantum cohomology cannot be semisimple, but its even part is a closed Frobenius subspace, and in principle it can be semisimple. In [5, Theorem 1.8.1], it was proved that if $H_{\text {quant }}^{\mathrm{ev}}(V)$ is generically semisimple, then $h^{p, q}(V)=0$ for $p+q \equiv 0 \bmod 2$, $p \neq q$. In the first section of this note we show that in this case $h^{p, q}(V)=0$ for $p+q \equiv 1 \bmod 2$ as well.

Thus, Theorem 1.8.1 of Bayer-Manin [5] can be strengthened in the following way: If the even quantum cohomology of a projective algebraic manifold $V$ is generically semisimple, then $V$ has no odd cohomology and is of Hodge-Tate type. In particular, for the 47 families of Fano threefolds (classified by V.A. Iskovskikh, Sh. Mori, and Sh. Mukai) with $b_{3}(V)>0$, the pure even quantum cohomology cannot be semisimple. This answers a question discussed in [6, p. 826].

The second section is devoted to a strengthening of a previously unpublished result of C. Hertling (a letter dated March 9, 2005, where it was stated for the pure even case). It shows that an analytic

\footnotetext{
${ }^{a}$ Institut für Mathematik, Universität Mannheim, A5, 6, 68131 Mannheim, Germany.

${ }^{b}$ Northwestern University, 633 Clark Street, Evanston, IL 60208, USA.

${ }^{c}$ Max-Planck-Institut für Mathematik, Vivatsgasse 7, 53111 Bonn, Germany.

${ }^{d}$ University of Edinburgh, King's Buildings, Mayfield Road, Edinburgh, EH9 3JZ, UK.

${ }^{e}$ University of California, 970 Evans Hall \#3840, Berkeley, CA 94720-3840, USA.

E-mail addresses: hertling@math.uni-mannheim.de (C. Hertling), manin@mpim-bonn.mpg.de (Yu. I. Manin), c.teleman@ed.ac.uk (C. Teleman).
} 
(or formal) supermanifold $M$ with a given supercommutative associative $\mathcal{O}_{M}$-bilinear multiplication on its tangent sheaf $\mathcal{T}_{M}$ is an $F$-(super)manifold in the sense of [12] if and only if its spectral cover, as an analytic subspace of the cotangent bundle $T_{M}^{*}$, is coisotropic of maximal dimension. This answers a question posed to Yu. Manin by V. Ginzburg.

\section{SEMISIMPLE QUANTUM COHOMOLOGY AND DUBROVIN'S CONJECTURE}

1.1. Notation. Let $V$ be a projective manifold over $\mathbb{C}$. We denote by $H_{\text {quant }}^{\mathrm{ev}}(V)$ its even quantum cohomology ring. As in [5] and [4], it is a topological commutative algebra. The multiplication in it (the classical cup product plus "quantum corrections") is denoted by o. The space $H^{\mathrm{ev}}(V)$ is embedded in it as a field of flat vector fields on the respective formal Frobenius manifold.

1.2. Proposition. If $H_{\mathrm{quant}}^{\mathrm{ev}}(V)$ is generically reduced, i.e., has no nilpotents at (the local ring of) the generic point, then $H^{\text {odd }}(V)=0$.

Proof. Assume that $H^{\text {odd }}(V) \neq 0$. Let $\Delta$ be a nonzero class of an odd dimension.

First, we have $\Delta \circ \Delta=0$. In fact, $\Delta \cup \Delta=0$, because the cup product is supercommutative. The quantum corrections vanish, because the correlators $\langle\ldots\rangle$ are also supercommutative in their arguments, so $\left\langle\Delta \Delta \Delta^{\prime} \ldots\right\rangle=0$. This follows from the fact that the quantum correlators come from the $S_{n}$-covariant maps $H^{*}(V)^{\otimes n} \rightarrow H^{*}\left(\bar{M}_{0, n}\right)$ induced by algebraic correspondences (push-forwards of virtual fundamental classes). On the source $S_{n}$ acts by permuting factors; the Koszul rule prescribes the relevant signs. On the target it renumbers points and hence leaves the fundamental class invariant.

Now, find another (odd) class $\Delta^{\prime}$ such that $g\left(\Delta, \Delta^{\prime}\right)=1$, where $g$ is the Poincare form. Then we have $1=g\left(\Delta, \Delta^{\prime}\right)=g\left(\Delta \circ \Delta^{\prime}, e\right)$, where $e$ is the identity in quantum cohomology. Hence $\Delta \circ \Delta^{\prime} \in H_{\text {quant }}^{\text {ev }}(V)$ must be generically nonzero. But its square is zero because of the first remark. This contradicts the generic absence of nilpotents in $H_{\text {quant }}^{\mathrm{ev}}(V)$.

1.3. Theorem. If the even quantum cohomology of a projective algebraic manifold $V$ is generically semisimple, then $V$ has no odd cohomology and is of Hodge-Tate type.

Proof. From the generic semisimplicity and Proposition 1.2 it follows that $h^{p, q}(V)=0$ for $p+q \equiv 1 \bmod 2$.

To prove that $h^{p, q}(V)=0$ for $p+q \equiv 0 \bmod 2$ and $p \neq q$, we reproduce a short reasoning from [5]. It compares the Lie algebra of Euler vector fields in the semisimple case and in the quantum cohomology case.

Firstly, in the semisimple case each Euler vector field must be of the form $E=d_{0} \sum_{i} u_{i} e_{i}+$ $\sum_{j} c_{j} e_{j}$, where $d_{0}$ is a constant (the weight of $E$, cf. $[13,14]$ ) and $\left(u_{i}\right)$ are (local) Dubrovin's canonical coordinates, that is, $e_{i}:=\partial / \partial u_{i}$ form a complete system of pairwise orthogonal idempotents in $H_{\text {quant }}^{*}(V)$. Moreover, $\left(c_{j}\right)$ are arbitrary constants.

From this explicit description it follows directly that if two Euler fields of nonzero weights commute, they are proportional.

On the other hand, if $h^{p, q}(V) \neq 0$ for some $p+q \equiv 0 \bmod 2$ and $p \neq q$, then $H_{\text {quant }}^{*}(V)$ admits two commuting and nonproportional Euler vector fields $E_{1}$ and $E_{2}$ of weight 1 . Namely, in the bihomogeneous (with respect to the $(p, q)$-grading) basis of flat vector fields $\Delta_{a} \in H^{p_{a}, q_{a}}(V)$, we can take

$$
E_{1}:=\sum_{a}\left(1-p_{a}\right) x_{a} \Delta_{a}+\sum_{p_{b}=q_{b}=1} r_{b} \Delta_{b}, \quad E_{2}:=\sum_{a}\left(1-q_{a}\right) x_{a} \Delta_{a}+\sum_{p_{b}=q_{b}=1} r_{b} \Delta_{b} .
$$

Here $\left(x_{a}\right)$ are dual flat coordinates, and $-K_{V}=c_{1}\left(\mathcal{T}_{V}\right)=\sum_{b} r_{b} \Delta_{b}$.

This completes the proof. 
1.4. Dubrovin's conjecture and related insights. In $[7$, p. 321] the problem of characterization of varieties $V$ with semisimple quantum cohomology was formulated explicitly. Dubrovin conjectured that only Fano varieties can have generically semisimple quantum cohomology. This was disproved by A. Bayer [4], who established that blowing up points on such a variety does not destroy semisimplicity. In particular, not only del Pezzo surfaces have semisimple quantum cohomology, but arbitrary blowups of $\mathbb{P}^{2}$ as well.

A. Bayer has later conjectured that the maximal length of a semiorthogonal decomposition of $D^{\mathrm{b}}(V)$ must coincide with the generic number of idempotents in $H_{\text {quant }}^{*}(V)$.

Combining the results of [4], of this note, and the further part of Dubrovin's conjecture stated on p. 322 of [7] (cf. also [21]), one can now guess that a necessary and sufficient condition for semisimplicity is that $V$ is of Hodge-Tate type and its bounded derived coherent category admits a full exceptional collection $\left(E_{i}\right)$. Moreover, after adjusting some arbitrary choices, in this case one should be able to identify the Stokes matrix of its second structure connection with the matrix $\left(\chi\left(E_{i}, E_{j}\right)\right)$.

This last statement is now checked, in particular, for three-dimensional Fano varieties with minimal cohomology in [10]. The reader can find there more details and explanations about the involvement of the vanishing cycles in the mirror Landau-Ginzburg model.

All these constructions reflect some facets of Kontsevich's homological mirror symmetry program. However, one should keep in mind that in this note we are concerned almost exclusively with a multiplication on the tangent bundle, i.e., with the structure of an $F$-manifold (see below). In order to invoke mirror symmetry, we also need to take into consideration a compatible flat metric. In quantum cohomology, it comes "for free" at the start; it is multiplication that requires a special construction. In various contexts relevant to mirror symmetry, the metric can be described implicitly by at least five different kinds of data, which we list here for the reader's convenience.

(a) Values of the diagonal coefficients of the flat metric $\sum_{i} \eta_{i}\left(d u_{i}\right)^{2}$ in canonical coordinates and values of their first derivatives $\eta_{i j}$ at a tame semisimple point. These are initial data for the second structure connection (cf. [13, II.3]).

(b) Monodromy data for the first structure connection and oscillating integrals for the deformed flat coordinates (cf. $[8,7,19]$ and the references therein).

(c) Choice of one of K. Saito's primitive forms.

(d) Choice of a filtration on the cohomology space of the Milnor fiber (M. Saito, cf. [11] and the references therein).

(e) Use of the semi-infinite Hodge structure. This is a refinement of (c), described by S. Barannikov $[2,3]$.

\section{F-GEOMETRY AND SYMPLECTIC GEOMETRY}

2.1. F-structure and Poisson structure. Manifolds $M$ considered in this section can be $C^{\infty}$, analytic, or formal, eventually with even and odd coordinates (supermanifolds). The ground field $K$ of characteristic zero is most often $\mathbb{C}$ or $\mathbb{R}$. Each such manifold, by definition, is endowed with the structure sheaf $\mathcal{O}_{M}$, which is a sheaf of (super)commutative $K$-algebras, and the tangent sheaf $\mathcal{T}_{M}$, which is a locally free $\mathcal{O}_{M}$-module of (super)rank equal to the (super)dimension of $M$. $\mathcal{T}_{M}$ acts on $\mathcal{O}_{M}$ by derivations and is a sheaf of Lie (super)algebras with an intrinsically defined Lie bracket $[$,$] .$

There is a classical notion of Poisson structure on $M$, which also endows $\mathcal{O}_{M}$ with a Lie bracket $\{$,$\} constrained by a well-known identity.$

Similarly, an $F$-structure on $M$ endows $\mathcal{T}_{M}$ with an extra operation: (super)commutative and associative $\mathcal{O}_{M}$-bilinear multiplication. We denote it always by $\circ$ and assume that it is endowed with an identity: an even vector field $e$. Then $\mathcal{O}_{M}$ is embedded in $\mathcal{T}_{M}$ as a subalgebra: $f \mapsto f e$. 
Given such a multiplication on the tangent sheaf, we can define its spectral cover $\widetilde{M}$, which is a closed ringed (super)subspace (generally not a submanifold) in the cotangent (super)manifold $T^{*} M$. In the Grothendieck language, it is simply the relative affine spectrum of the sheaf of algebras $\left(\mathcal{T}_{M}, \circ\right)$ on $M$.

More precisely, consider $\operatorname{Symm}_{\mathcal{O}_{M}}\left(\mathcal{T}_{M}\right)$ as the sheaf of algebras of those functions on the cotangent (super)space $T_{M}^{*}$ that are polynomial along the fibers of the projection $T_{M}^{*} \rightarrow M$. The multiplication in this sheaf will be denoted by a dot. For example, for two local vector fields $X, Y \in \mathcal{T}_{M}(U), X \cdot Y$ denotes their product as an element of $\operatorname{Symm}_{\mathcal{O}_{M}}^{2}\left(\mathcal{T}_{M}\right)$.

Consider the canonical surjective morphism of sheaves of $\mathcal{O}_{M}$-algebras

$$
\operatorname{Symm}_{\mathcal{O}_{M}}\left(\mathcal{T}_{M}\right) \rightarrow\left(\mathcal{T}_{M}, \circ\right)
$$

sending, say, $X \cdot Y$ to $X \circ Y$. Denote its kernel by $J(M, \circ)$, and let $\widetilde{M}$ be defined by the sheaf of ideals $J(M, \circ)$.

The spectral cover $\widetilde{M} \rightarrow M$ is flat, because $\mathcal{T}_{M}$ is locally free.

Now we will describe the structure identities imposed onto $\{$,$\} on \mathcal{O}_{M}$ and onto $\circ$ on $\mathcal{T}_{M}$. To this end, recall the notion of the Poisson tensor. Let generally $A$ be a $K$-linear superspace (or a sheaf of superspaces) endowed with a $K$-bilinear multiplication and a $K$-bilinear Lie bracket [,]. Then for any $a, b, c \in A$ put

$$
P_{a}(b, c):=[a, b c]-[a, b] c-(-1)^{a b} b[a, c] .
$$

(From here on, $(-1)^{a b}$ and similar notation refer to the sign occurring in the superalgebra when the two neighboring elements get permuted.)

This tensor will be written for $A=\left(\mathcal{O}_{M}, \cdot,\{\},\right)$ in the case of the Poisson structure and for $A=\left(\mathcal{T}_{M}, \circ,[],\right)$ in the case of an $F$-structure.

We will now present parallel lists of basic properties of Poisson manifolds and $F$-manifolds.

2.2. Poisson (super)manifolds. (i) $P$ Structure identity: for all local functions $f, g$, and $h$ on $M$

$$
P_{f}(g, h) \equiv 0 .
$$

(ii) ${ }_{P}$ Each local function $f$ on $M$ becomes a local vector field $X_{f}$ (of the same parity as $f$ ) on $M$ via $X_{f}(g):=\{f, g\}$.

This is a reformulation of $(2.2)$.

(iii) $)_{P}$ Maximally nondegenerate case: symplectic structure. There exist local canonical coordinates $\left(q_{i}, p_{i}\right)$ such that for any $f$ and $g$

$$
\{f, g\}=\sum_{i=1}^{n}\left(\partial_{q_{i}} f \partial_{p_{i}} g-\partial_{q_{i}} g \partial_{p_{i}} f\right) .
$$

Thus, locally all symplectic manifolds of the same dimension are isomorphic. The local group of symplectomorphisms is, however, infinite-dimensional.

2.3. F-manifolds. (i) $)_{F}$ Structure identity: for all local vector fields $X, Y, Z$, and $U$

$$
P_{X \circ Y}(Z, U)-X \circ P_{Y}(Z, U)-(-1)^{X Y} Y \circ P_{X}(Z, U)=0 .
$$

(ii) $)_{F}$ Each local vector field on $M$ becomes a local function on the spectral cover $\widetilde{M}$ of $M$.

As we already mentioned, generally $\widetilde{M}$ is not a (super)manifold. In the pure even case this often happens because of nilpotents in $\mathcal{O}_{\widetilde{M}}$ and/or singularities. In the presence of odd coordinates on $M$ 
nilpotents are always present, but typically they cannot form an exterior algebra over functions of even coordinates because ranks do not match.

For more details on $F$-structure, see [14-17].

A theorem due to Hertling describes certain important cases when $\widetilde{M}$ is a manifold.

(iii) $F$ Maximally nondegenerate case: semisimple F-manifolds. $\widetilde{M}$ will be a manifold and even an unramified covering of $M$ in the appropriate "maximally nondegenerate case," namely, when $M$ is pure even, and locally $\left(\mathcal{T}_{M}, \circ\right)$ is isomorphic to $\left(\mathcal{O}_{M}^{d}\right)$ as an algebra, $d=\operatorname{dim} M$.

In this case there exist local canonical coordinates $\left(u_{a}\right)$ (Dubrovin's coordinates) such that the respective vector fields $\partial_{a}:=\partial / \partial_{a}$ are orthogonal idempotents:

$$
\partial_{a} \circ \partial_{b}=\delta_{a b} \partial_{a} .
$$

Thus, locally all semisimple $F$-manifolds of the same dimension are isomorphic. Local automorphisms of an $F$-semisimple structure are generated by renumberings and shifts of canonical coordinates:

$$
u_{a} \mapsto u_{\sigma(a)}+c_{a},
$$

so that this structure is more rigid than the symplectic one.

2.4. Spectral cover as a subspace in a symplectic supermanifold. There is a structure of a sheaf of Lie algebras on $\operatorname{Symm}_{\mathcal{O}_{M}}\left(\mathcal{T}_{M}\right)$. It is given by the Poisson brackets $\{$,$\} with respect to$ the canonical (super)symplectic structure on $T_{M}^{*}$.

It is easy to check that the ideal $J=J(M, \circ) \subset \operatorname{Symm}_{\mathcal{O}_{M}}\left(\mathcal{T}_{M}\right)$ defining $\widetilde{M}$ in this sheaf of supercommutative algebras is generated by all expressions

$$
e-1, \quad X \circ Y-X \cdot Y, \quad X, Y \in \mathcal{T}_{M} .
$$

2.5. Theorem. The multiplication o satisfies the structure identity of F-manifolds (2.3) if and only if the ideal $J(M, \circ)$ is stable with respect to the Poisson brackets.

Proof. From (2.2), one easily infers that the stability of an ideal in a Poisson algebra with respect to the brackets can be checked on any system of generators of this ideal. In our case we choose (2.4).

Clearly, $\{e-1, e-1\}=0$.

If $X$ and $Y$ are local vector fields, then $\{X, Y\}=[X, Y]$.

We will establish by a direct computation that for all $X, Y, Z$, and $W$ as above,

$$
\begin{aligned}
& \{X \circ Y-X \cdot Y, Z \circ W-Z \cdot W\} \\
& \quad \equiv P_{X \circ Y}(Z, W)-X \circ P_{Y}(Z, W)-(-1)^{X Y} Y \circ P_{X}(Z, W) \bmod J(M, \circ)
\end{aligned}
$$

and

$$
\{e-1, X \circ Y-X \cdot Y\}=[e, X \circ Y]-X \cdot[e, Y]-[e, X] \cdot Y .
$$

Assume that this is done. From (2.5) and (2.6) it follows that if (2.3) holds, then $J(M, 0)$ is stable with respect to the Poisson brackets. For (2.6), one uses the identity $[e, X \circ Y]=X \circ[e, Y]+$ $[e, X] \circ Y$, which follows from (2.3) by choosing $X=Y=e$ and renaming $Z$ and $U$.

Conversely, if $J(M, \circ)$ is stable with respect to the brackets, then the right-hand side of $(2.5)$ must belong to $J(M, \circ)$. But it lies in the degree 1 part of the symmetric algebra of $\mathcal{T}_{M}$, which projects onto $\mathcal{T}_{M}$. Hence it must vanish, and as a result, the right-hand side of (2.6) must belong to $J(M, \circ)$ as well.

It remains to check (2.5) and (2.6). We will briefly indicate how to do this, restricting ourselves to the clumsier case $(2.5)$. 
First of all, the right-hand side of (2.5) can be rewritten as follows:

$$
\begin{aligned}
P_{X \circ Y}(Z, W) & -X \circ P_{Y}(Z, W)-(-1)^{X Y} Y \circ P_{X}(Z, W) \\
= & {[X \circ Y, Z \circ W]-[X \circ Y, Z] \circ W-(-1)^{(X+Y) Z} Z \circ[X \circ Y, W]-X \circ[Y, Z \circ W] } \\
& -(-1)^{X Y} Y \circ[X, Z \circ W]+X \circ[Y, Z] \circ W+(-1)^{Y Z} X \circ Z \circ[Y, W] \\
& +(-1)^{X Y} Y \circ[X, Z] \circ W+(-1)^{X(Y+Z)} Y \circ Z \circ[X, W] .
\end{aligned}
$$

It turns out that $(2.7)$ is in fact a tensor, i.e., is $\mathcal{O}_{M}$-polylinear in $X, Y, Z$, and $W$. See $[16,17]$ for a discussion and operadic generalizations of the condition of its vanishing.

In our context, this formula is convenient, because a straightforward decomposition of the lefthand side of (2.5) into Poisson monomials (constructed using two operations) gives exactly the same list of monomials as in (2.7) modulo $J(M, \circ)$, with the same signs.

Here are samples of calculations.

The first term $\{X \circ Y, Z \circ W\}$ on the left-hand side of (2.5) coincides with the first term in (2.7).

Using the Poisson identity (2.2), we find further

$$
-\{X \circ Y, Z \cdot W\}=-\{X \circ Y, Z\} \cdot W-(-1)^{(X+Y) Z} Z \cdot\{X \circ Y, W\} .
$$

Modulo $J(M, \circ)$, this can be replaced by

$$
-[X \circ Y, Z] \circ W-(-1)^{(X+Y) Z} Z \circ[X \circ Y, W],
$$

which corresponds to the second and third terms of $(2.7)$.

We leave the rest as an exercise to the reader.

2.5.1. Reduced spectral cover. Contrary to what might be expected, the condition

$$
\{J(M, \circ), J(M, \circ)\} \subset J(M, \circ)
$$

does not imply the respective condition for the radical of $J(M, \circ)$ even in the pure even case. This means that $\widetilde{M}_{\text {red }}$ need not be a Lagrange subvariety, even if it comes from an $F$-manifold.

This can be shown with the following explicit examples.

We will construct two families of everywhere indecomposable (see Subsection 2.6 below) $F$-manifolds in terms of the ideals $J$ defining (nonreduced) subspaces $\widetilde{M} \subset T^{*} M$. In order to give rise to $F$-manifolds with $\pi: \widetilde{M} \rightarrow M$ as their spectral cover, they have to satisfy the following conditions:

(a) The projection $\widetilde{M} \rightarrow M$ is flat of degree $n=\operatorname{dim} M$ and the canonical map $\mathcal{T}_{M} \rightarrow \pi_{*}\left(\mathcal{O}_{\widetilde{M}}\right)$ is an isomorphism.

To check this by direct calculations, we will choose (pure even) local coordinates $\left(t_{1}, \ldots, t_{n}\right)$ on $M$ in such a way that $e=\partial / \partial t_{1}$. By $\left(y_{1}, \ldots, y_{n}\right)$ we will denote the conjugate coordinates along the fibers of $T^{*}(M)$.

(b) $\{J, J\} \subset J$.

We will see that in these examples

$$
\{\sqrt{J}, \sqrt{J}\} \not \subset \sqrt{J} .
$$

2.5.2. The first family. Here we put

$$
J=\left(y_{1}-1,\left(y_{i}-\rho_{i}\right)\left(y_{j}-\rho_{j}\right)\right),
$$

with $\rho_{1}=1$ and $\rho_{i} \in \mathcal{O}_{M}$ for $i \geq 2$ such that $\partial_{1} \rho_{i}=0$. Clearly, (a) and (b) are satisfied. The radical of $J$ is

$$
\sqrt{J}=\left(y_{1}-1, y_{2}-\rho_{2}, \ldots, y_{n}-\rho_{n}\right) .
$$


We have $\{\sqrt{J}, \sqrt{J}\} \not \subset \sqrt{J}$ if

$$
\partial_{i} \rho_{j} \neq \partial_{j} \rho_{i} \quad \text { for some } i, j \geq 2 \text { with } i \neq j \text {. }
$$

The algebra $T_{t} M$ at any point $t \in M$ is isomorphic to $\mathbb{C}\left[x_{1}, \ldots, x_{n-1}\right] /\left(x_{i} x_{j}\right)$.

2.5.3. The second family. Here we put for any $n \geq 3$

$$
J=\left(y_{1}-1,\left(y_{2}-\rho_{2}\right)^{2},\left(y_{2}-\rho_{2}\right) \cdot y_{3}, y_{3}^{n-1}, y_{4}-y_{3}^{2}, y_{5}-y_{3}^{3}, \ldots, y_{n}-y_{3}^{n-2}\right),
$$

with

$$
\rho_{2}(y, t)=t_{3} y_{1}+\sum_{k=3}^{n-1}(k-1) t_{k+1} \cdot y_{k} .
$$

Now, (a) is rather obvious, but checking (b) requires a calculation, which we omit. The radical of $J$ is

$$
\sqrt{J}=\left(y_{1}-1, y_{2}-t_{3} \cdot y_{1}, y_{3}, y_{4}, y_{5}, \ldots, y_{n}\right)
$$

The algebra $T_{t} M$ at any point $t \in M$ is isomorphic to $\mathbb{C}\left[x_{2}, x_{3}\right] /\left(x_{2}^{2}, x_{2} x_{3}, x_{3}^{n-1}\right)$.

We will now explain in which context the considerations of this section can be related to the problems arising in the study of semisimple quantum cohomology.

2.6. Hertling's local decomposition theorem. For any point $x$ of a pure even $F$-manifold $M$, the tangent space $T_{x} M$ is endowed with the structure of a $K$-algebra. This $K$-algebra can be represented as a direct sum of local $K$-algebras. The decomposition is unique in the following sense: the set of pairwise orthogonal idempotent tangent vectors determining it is well defined.

C. Hertling has shown that this decomposition extends to a neighborhood of $x$.

More precisely, define the sum of two $F$-manifolds:

$$
\left(M_{1}, \circ_{1}, e_{1}\right) \oplus\left(M_{2}, \circ_{2}, e_{2}\right):=\left(M_{1} \times M_{2}, \circ_{1} \boxplus \circ_{2}, e_{1} \boxplus e_{2}\right) .
$$

A manifold is called indecomposable if it cannot be represented as a sum in a nontrivial way.

2.6.1. Theorem. Every germ $(M, x)$ of a complex analytic $F$-manifold decomposes into a direct sum of indecomposable germs such that, for each summand, the tangent algebra at $x$ is a local algebra.

This decomposition is unique in the following sense: the set of pairwise orthogonal idempotent vector fields determining it is well defined.

For a proof, see [11, Theorem 2.11].

Furthermore, we have [11, Theorems 5.3 and 5.6]:

2.7. Theorem. (i) The spectral cover space $\widetilde{M}$ of the F-structure on the germ of the unfolding space of an isolated hypersurface singularity is smooth.

(ii) Conversely, let $M$ be an irreducible germ of a generically semisimple F-manifold with the smooth spectral cover $\widetilde{M}$. Then it is (isomorphic to) the germ of the unfolding space of an isolated hypersurface singularity. Moreover, any isomorphism of germs of such unfolding spaces compatible with their F-structure comes from a stable right equivalence of the germs of the respective singularities.

Recall that the stable right equivalence is generated by adding sums of squares of coordinates and making invertible analytic coordinate changes. 
In view of this result, it would be important to understand the following

2.8. Problem. Characterize those varieties $V$ for which the quantum cohomology Frobenius spaces $H_{\text {quant }}^{*}(V)$ have smooth spectral covers.

Theorem 2.7 produces a weak version of the Landau-Ginzburg model for such manifolds and thus gives a partial solution of the mirror problem for them: compare with a much more detailed pictures in $[1,18]$.

\section{ACKNOWLEDGMENTS}

We are grateful to Arend Bayer for illuminating comments on Proposition 1.2 and for sharing with us his version of Dubrovin's conjecture.

\section{REFERENCES}

1. D. Auroux, L. Katzarkov, and D. Orlov, "Mirror Symmetry for Del Pezzo Surfaces: Vanishing Cycles and Coherent Sheaves," Invent. Math. 166 (3), 537-582 (2006); arXiv: math/0506166.

2. S. Barannikov, "Semi-infinite Hodge Structures and Mirror Symmetry for Projective Spaces," arXiv: math/0010157.

3. S. Barannikov, "Semi-infinite Variations of Hodge Structures and Integrable Hierarchies of KdV Type," Int. Math. Res. Not., No. 19, 973-990 (2002); arXiv: math/0108148.

4. A. Bayer, "Semisimple Quantum Cohomology and Blowups," Int. Math. Res. Not., No. 40, 2069-2083 (2004); arXiv: math/0403260.

5. A. Bayer and Yu. Manin, "(Semi)simple Exercises in Quantum Cohomology", in Proc. Fano Conf., Torino, Italy, 2002 (Univ. Torino, Torino, 2004), pp. 143-173; arXiv: math/0103164.

6. G. Ciolli, "On the Quantum Cohomology of Some Fano Threefolds and a Conjecture of Dubrovin," Int. J. Math. 16 (8), 823-839 (2005); arXiv: math/0403300.

7. B. Dubrovin, "Geometry and Analytic Theory of Frobenius Manifolds," in Proc. Int. Congr. Math., Berlin, 1998 (Univ. Bielefeld, Bielefeld, 1998), Vol. 2, pp. 315-326.

8. A. Givental, "A Mirror Theorem for Toric Complete Intersections," in Topological Field Theory, Primitive Forms and Related Topics (Birkhäuser, Boston, 1998), Prog. Math. 160, pp. 141-175.

9. V. V. Golyshev, "Riemann-Roch Variations," Izv. Ross. Akad. Nauk, Ser. Mat. 65 (5), 3-32 (2001) [Izv. Math. 65, 853-881 (2001)].

10. V. Golyshev, "A Remark on Minimal Fano Threefolds," arXiv: 0803.0031.

11. C. Hertling, Frobenius Manifolds and Moduli Spaces for Singularities (Cambridge Univ. Press, Cambridge, 2002).

12. C. Hertling and Yu. Manin, "Weak Frobenius Manifolds," Int. Math. Res. Not., No. 6, 277-286 (1999); arXiv: math/9810132.

13. Yu. I. Manin, Frobenius Manifolds, Quantum Cohomology, and Moduli Spaces (Am. Math. Soc., Providence, RI, 1999), AMS Colloq. Publ. 47.

14. Yu. I. Manin, "Manifolds with Multiplication on the Tangent Sheaf," Rend. Mat. Appl., Ser. 7, 26, 69-85 (2006); arXiv: math.AG/0502578.

15. Yu. I. Manin, "F-Manifolds with Flat Structure and Dubrovin's Duality," Adv. Math. 198, 5-26 (2005) (M. Artin's Fest).

16. S. A. Merkulov, "Operads, Deformation Theory and F-Manifolds," in Frobenius Manifolds: Quantum Cohomology and Singularities, Ed. by C. Hertling and M. Marcolli (Vieweg, Wiesbaden, 2004), pp. 213-251; arXiv: math.AG/0210478.

17. S. A. Merkulov, "PROP Profile of Poisson Geometry," Commun. Math. Phys. 262 (1), 117-135 (2006); arXiv: math.AG/0401034.

18. D. O. Orlov, "Derived Category of Coherent Sheaves and Motives," Usp. Mat. Nauk 60 (6), 231-232 (2005) [Russ. Math. Surv. 60, 1242-1244 (2005)]; arXiv: math/0512620.

19. C. Sabbah, Isomonodromic Deformations and Frobenius Manifolds (Springer, Berlin, 2007).

20. C. Teleman, "The Structure of 2D Semi-simple Field Theories," arXiv: 0712.0160.

21. E. Zaslow, "Solitons and Helices: The Search for a Math-Physics Bridge," Commun. Math. Phys. 175 (2), 337-375 (1996). 